\title{
Cast On Cast Off II
}

\section{Li-Fen Anny Chang, University of North Texas, USA}

Keywords: Knitting, draping, hand-stitching, zero-waste

As a designer and an educator, I am constantly searching for new sources of inspiration. The fashion world moves forward relentlessly, and if you cannot keep up, you will get left behind. Consider knitting, an old technique of producing fabric from strands of yarn, it is an art form often overlooked or considered outdated. However, as a fashion designer, investigating and understanding the technique of knitting posed an interesting opportunity to fully integrate the design process from fiber to finished garment. Therefore, I sought an opportunity to extensively study basic machine knitting to expand my personal creative work while supporting the work of my students. Through a connection, I was able to learn hand driven machine knitting from Dr. Yu, a professor in the Department of Textiles and Clothing at Fu Jen Catholic University in Taipei, Taiwan. The course included topics such as knitting terminology, machine basic operations, and techniques. Basic knitting techniques that were taught included single jersey, all-knit, rib, tubular, Milano, cardigan, pleating, and ottoman stitch using a seven-gauge hand driven flat double bed knitting machine (Spencer, 2010). Additionally, I learned to knit cording using a six-needle cord knitting machine with $1 / 4$ " diameter finish. Cords were knitted in different colors to be used for accent or function in the designs as needed (Figure 1).

During the study, through trial and error, I learned to test yarn elasticity and durability to prevent time wasted on aged or brittle yarns which are prone to breakage during the knitting process. For sustainability considerations, failed knitting pieces were saved to be unraveled and reused for later knitting or hand stitching (Figure 2). Due to the lack of knitting equipment available at my institution, I knitted several pieces in various sizes to design garments after my return to the United State. Design titled "Cast On Cast Off II" is the second garment created using the knitting pieces I created during my time with the Department of Textiles and Clothing at $\mathrm{Fu}$ Jen Catholic University.

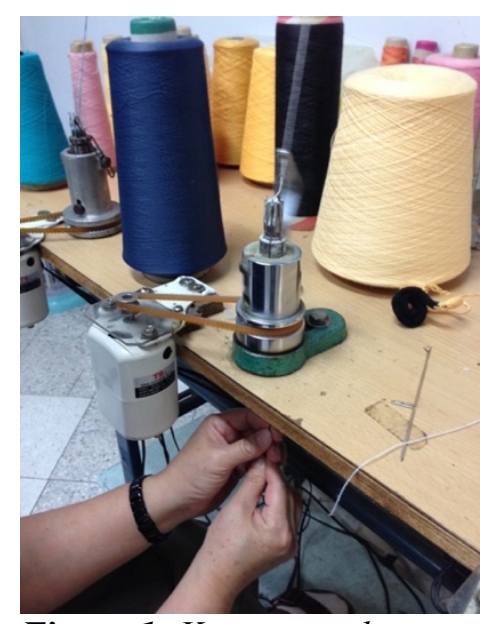

Figure 1: Knitting with sixneedle cord knitting machine

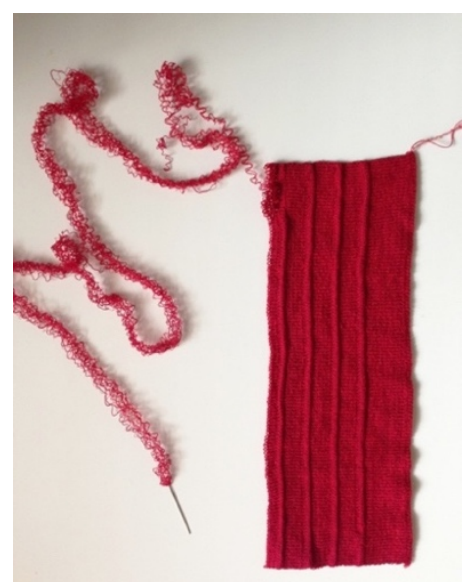

Figure 2: Deconstructed scrap piece used for hand stitching

Page 1 of 3

(c) 2020 The author(s). Published under a Creative Commons Attribution License (https://creativecommons.org/licenses/by/4.0/), which permits unrestricted use, distribution, and reproduction in any medium, provided the original work is properly cited.

ITAA Proceedings, \#77 - https: / /itaaonline.org 
The purpose of the design was to create a cocktail dress using an unconventional material- specifically yarn. The challenge of the design was to create a cocktail dress by assembling existing knitting pieces in a zero-waste manner. Using draping techniques, knits were first laid on the dress form to draw the style lines and design (XXXX, 2016). After experimenting the layout, three knits were chosen for the main body of the dress including one blue 17" x 27" with knitting structure of all-knit, one blue 6" x 38 " with knitting structure of ottoman stitch, and one yellow 15" x 37 $1 / 2$ " also with knitting structure of the ottoman stitch. These two knitting pieces were knitted in different widths but with the same stitch density, same number of course and wale per inch, and feature of ottoman stitches. The ottoman stitch was created by using the front bed needles and knitting for five courses. This generates the piping look (Figure 3).

Once the design was preliminarily established on the dress form, I used hand slip stitching to connect pieces together and finish raw edges. The left seam was left unstitched for eight inches to allow for an armhole; then, the seam was further stitched to form a built-up neckline. A yellow cord

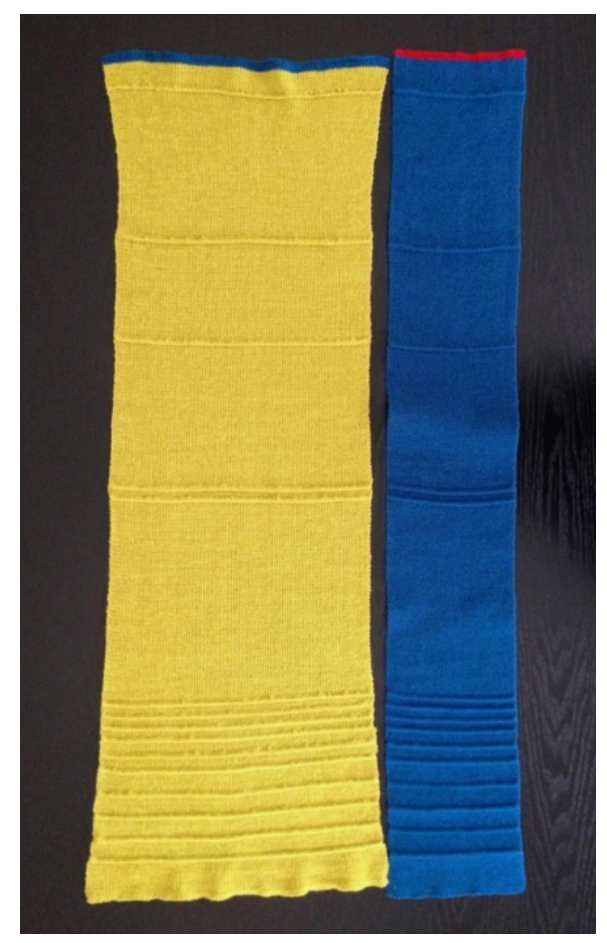

Figure 3: Matching ottoman stitches was set along the inside of the armhole to prevent from stretching. Because of its rectangular shape, the blue all-knit panel on the right back neck was loose, so I folded a pleat for fit and set a blue cord to secure the pleat permanently. Similarly, for the upper left back, three ottoman stitches were folded at one end to conform the body, and a blue cord was set to secure the folds. Proceeded by the blue cord, the red cords were added for function and design accent. The first red cord was set starting at the front blue hem to the right armhole, connecting the left back neck to form a neckline, and continuing back to the front hem. The second red cord was set doubly at the right armhole to serve as right shoulder strap for carrying the dress. The third red cord was set doubly and hung loosely for design accent starting at the right front armhole to the back. The fourth red cord was also set doubly and intentionally set loosely. Intended to not only keep the red neck cord in place, the soft cord lays freely resting on the neck to create a delicate and adoring look. Each end of cord was clean finished by hand slip stitching. These six red cords passed the layers of pleat on right back shoulder, secured by hand stitches, and formed a knot with tassels as the focal point.

The art of knitting using precise machinery to bind individual threads into a single entity cannot be learned alone. After returning to my own institution, the lack of knitting equipment posed a challenge for my practicing, progressing, and perfecting of the techniques I learned at the $\mathrm{Fu}$ Jen Catholic University. Nevertheless, I undertook the challenge, utilized my new understanding 
in knitting, and created a second knit design. "Cast On Cast Off II" features bias drapes of yellow and blue ottoman stitch pieces with a blue all-knit piece and red cords strap forming a knot with tassels on the back. The yarn colors of red, yellow, and blue were selected for the contrast effect. The content of yarns is $100 \%$ acrylic with characteristics of durability, easy care, and softness.

\section{References:}

Spencer, D. J. (2010). Knitting technology: a comprehensive handbook and practical guide (3 ed.). Cambridge, England: Woodhead.

XXXX, (2016). XXXX. Proceedings of the International Textile and Apparel Association Annual Conference Proceedings, XX. Retrieved from XXXX. 

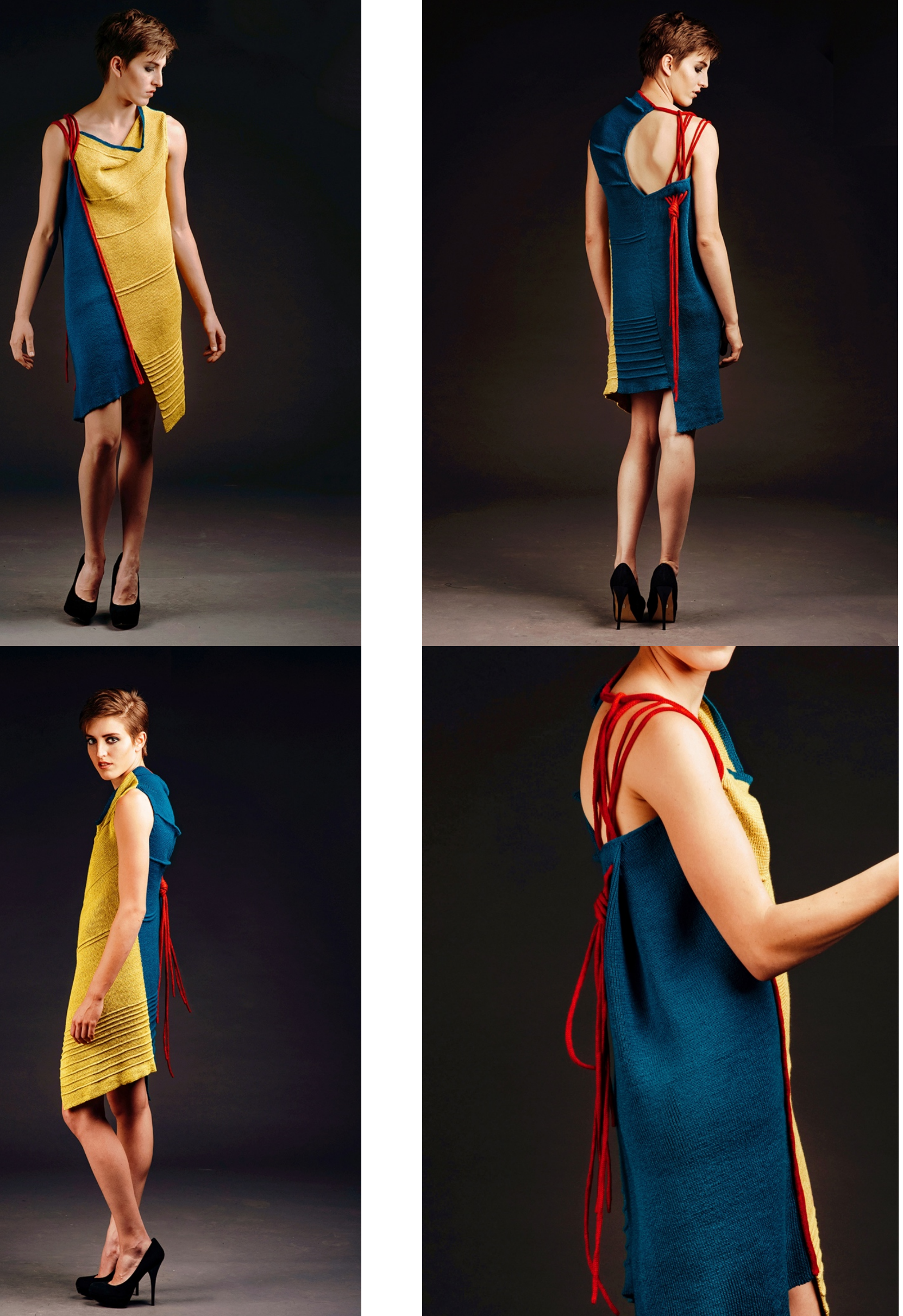